\title{
Vegetal Metabolomics to Seeds of Galium Aparine
}

\section{Monica Butnariu ${ }^{1 *}$, lonel Samfira²}

${ }^{1}$ Discipline of Chemistry and Vegetal Biochemistry, Banat's University of Agricultural Sciences and Veterinary Medicine "Regele Mihai I al Romaine" from Timisoara, 300645, Calea Aradului 119, Timis, Romania

${ }^{2}$ Discipline of Grassland and Fodder plants culture, Banat's University of Agricultural Sciences and Veterinary Medicine "Regele Mihai I al Romaine" from Timisoara, 300645, Calea Aradului 119, Timis, Romania

\section{Editorial}

Gallium aparine is an annual herbaceous plant that grows on road sides, pastures and uncultivated places, mostly wetly, at altitudes up to $2500 \mathrm{~m}$ (Figure 1). It can be found in landscape of Europe, Asia and North America; belongs to family Rubiaceae or Rubiacee. The plant grows to $1 \mathrm{~m}$ high, has strains creeping and hard endowed in place with small hooks. The flowers are hermaphrodite, with white petals in shape of small stars and grains are round, small and present numerous sticky hairs that stick easily to animal fur, they are why plant is called "sticky". It is very simple plant propagation by seed dispersal elsewhere. Gallium name comes from Greek word meaning milk and refers to fact that plant can help to coagulate milk and then preparation of cheese.

The sticky herb is used for internal and external use, harvested its parts air-leaves and branches; which are put to dry in a well-ventilated, clean and shaded [1]. The seeds of Galium aparine have a high content of bioactive compounds (vitamins, phenolic compounds, carotenoids) important in human health and nutrition. Chemical ingredients of $G$. aparine include: iridoid glycosides such as asperulosidic acid and 10deacetylasperulosidic acid, asperuloside, aucubin and monotropein, alkaloids such as caffeine, phenolics such as phenolic acids, anthraquinone derivatives such as aldehyde norda mnacanthal $(1,3-$ dihydroxy-anthraquinone-2-al), flavonoids, coumarins, organic acids such as citric acid and a red dye. Sticky can combat staphylococcal infections. It is beneficial for cleansing, purifying and detoxifying body.

Sticky is a species of Goldenrod white, less known to consumers for natural products that give off a very sticky substance, so he received this name popular [2]. From resulting sticky powder is used in aerial parts of plant, in course of 30 days. Sticky is one of most effective remedies against staphylococcal infections; has a strong anti-inflammatory, boosts immunity, fights psoriasis, cystitis, kidney stones, seborrhoea dermatitis, fluid retention, use compresses to remove staph infections of skin [3].

Among bioactive compounds, seeds of G. aparine contain a variety of phenolic compounds and carotenoids that can be considered biomarkers quality products and authenticity. The purpose was to apply comparative screening of varieties of seeds sticky herb carotenoids and polyphenols domestic use as biomarkers of biological authentication. In this way, were analysed twelve varieties of seeds stickys, of spontaneous-unapproved, using advanced analytical techniques such as UV-VIS spectroscopy, high performance liquid chromatography (HPLC with UV detection/PDA) and FTIR spectroscopy, interpreted

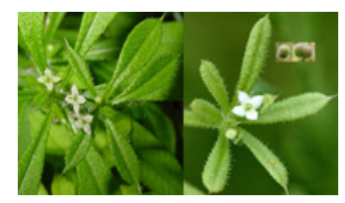

Figure 1: Gallium aparine. by chemometric analysis of PCA (Principal Component analysis) and CA (Cluster analysis) [4].

Analysis of metabolic profile of seeds of G. aparine was of "nontarget", which were made qualitative assessment of overall impression of biomarkers carotenoids and polyphenols [5].

Spectroscopy is an experimental method that measures and interprets this interaction. Although electromagnetic radiation used in common in nature, it is found that physico-chemical effects of substance varies depending on radiation energy, nature and structure of product. The interaction of one part translates through absorption spectra, emission or scattering of electromagnetic waves, on other hand transient or permanent changes in optical, electrical, magnetic, thermal, chemical substance composed of atoms, molecules, ions or other chemical species. Spectroscopy was originally study of interaction between radiation and matter as a function of wavelength $(\lambda)[6]$.

Spectrum refers to use of dispersion of visible light according to its wavelength, such as through a prism. Later concept was expanded greatly to comprise any quantitative determination based on either wavelength or frequency. Thus, it also may relate response to alternating field or a variable frequency $(v)$.

Another extension of scope of spectroscopy is adding a variable energy $(E)$, once it was realized relationship $E=h v$ incident photon ( $h$ is Planck's constant). Spectroscopy/spectrometry is often used in physical and analytical chemistry for identification of substances through spectrum emitted or absorbed by them. Ultraviolet and Visible Spectroscopy in (UV-Vis) is an analytical method applied in structure of organic compounds are conjugated multiple bonds (unsaturated and aromatic compounds) [7].

Although this method can be applied to a relatively small type of organic compounds, however it has advantage of selectivity absorption $\mathrm{UV}-\mathrm{Vis}$ by polyenic systems, which enable an easy presence of these systems in complex molecular structures. Infrared absorption spectroscopy is one of most important techniques used in investigation of material (which is both crystalline and non-crystalline state), molecular applicable to systems in any state of aggregation used for:

*Corresponding author: Monica Butnariu, Discipline of Chemistry and Vegetal Biochemistry, Banat's University of Agricultural Sciences and Veterinary Medicine "Regele Mihai I al Romaine" from Timisoara, 300645, Calea Aradului 119, Timis, Romania, Tel: +40-0-256-277-464; E-mail: monicabutnariu@yahoo.com; monica_butnariu@usab-tm.ro

Received November 25, 2013; Accepted November 28, 2013; Published December 05, 2013

Citation: Butnariu M, Samfira I (2013) Vegetal Metabolomics to Seeds of Galium Aparine. J Bioequiv Availab 5: e45. doi:10.4172/jbb.10000e45

Copyright: (C) 2013 Butnariu M, et al. This is an open-access article distributed under the terms of the Creative Commons Attribution License, which permits unrestricted use, distribution, and reproduction in any medium, provided the original author and source are credited. 
- Identifying all types of organic and inorganic compounds;

- Determination of functional groups in organic materials;

- Determining composition of molecular surface;

- Identification of chromatographic;

- Quantification of compounds in mixture;

- Non-destructive method;

- Determination of molecular structures (structural isomers) and stereochemistry (geometric isomers);

- Determination of molecular orientations (polymer solutions).

Raman spectroscopy complements IR spectroscopy. IR absorption spectroscopy and Raman photons used to determine modes of vibration of probe. Using Raman spectroscopy to larger scale structure analysis of vitreous is more recent than IR spectroscopy. The Raman effects is caused by change in electronic charge distribution under influence of electromagnetic radiation applied to variation of load distribution and not nuclear molecule, therefore Raman spectroscopy working with far superior luminous radiant frequency molecular vibrations. The process underlying Raman effects is diffusion of substance of an intense beam of light energy incident. The light is diffused matter in all directions.

By analysing light scatter was found that in addition to radiation and that incident frequency (Rayleigh scattering) appear lower frequency radiation $\left(v_{\text {exc }}-\Delta v\right)$ or higher $\left(v_{\text {exc }}+\Delta v\right)$ than incident (Stokes and anti-Stokes Raman-scattering). $\Delta v$ frequencies coincide with frequencies of vibration of molecules making up substance diffused. The information obtained is vibration frequencies of groups of atoms corresponding to fundamental electrical state [8]. In relation to IR spectra, spectra produced by Raman spectroscopy having following characteristic advantages:

- Emphasized highs are generally well defined, limited in number and often polarized;

- Are simple and depend strongly on composition;

- They have a low sensitivity to surface contamination, and moisture content;

- Samples were larger, to measure effects of volume.

A high temperature measurement is easy. High Performance Liquid Chromatography (HPLC) is a method for qualitative analysis of biochemistry and analytical chemistry used to separate, identify and quantify compounds. Using a column loaded with different materials (stationary phase), a pump that draws mobile step (s) through column and a detector showing retention time of molecules. The retention time depends on interaction between stationary phase, molecules to be analyzed, and solvents used.

The sample to be analyzed is introduced into small volume flow of mobile phase. Moving analyte through column is slowed by presence of chemical or physical interactions in stationary phase, which went to column. A better refining HPLC method results in possibility to vary composition of mobile phase during analysis; this is known as gradient elution. The bio compounds were isolated by ethyl acetate-soluble part of $95 \%$ ethanol extract seeds of G. aparine, and their chemical structures were identified as vanillic acid, gallic acid, 1-(4-hydroxyphenyl)-ethanone, 3,4-dihydroxybenzoic acid, p-hydroxycinnamic acid, 4-hydroxytruxillic acid. At same time were determined total concentration of carotenoids and polyphenols. On average, seeds of $G$. aparine analyzed carotenoid concentrations were $50 \mathrm{mg} / 100 \mathrm{~g}-700 \mathrm{mg} / 100 \mathrm{~g}$ dry substance total phenolic compounds, ranging from one to other biotype.

According to data obtained by HPLC analysis, main types of carotenoids were retinol, alpha-tocopherol, lutein /zeaxanthin, alphacarotene, beta-carotene, beta-cryptoxanthin, lutein and lycopene, free carotenids and zeaxanthin esters, followed by-esters of lutein and $\beta$-cryptoxanthin ones. Phenolic compounds were identified in class flavonol glycosides were quantified: I3-neohespiridoside, I3glucoside), I3-rhamnosylglucoside), I3-sophoroside-7-rhamnoside and free isorhamnetin. Infra-red spectroscopy (IR) is most suitable method for identifying presence of polar functional groups in molecular structure of organic compounds [9]. Infrared radiation (IR) is that portion of electromagnetic spectrum between visible and microwave region, characterized in that wavelength is of order of $10^{-5} \mathrm{~m}$. For recording IR spectra used to determine structure using only organic compounds are IR means, which contains wavelength located in region of $2.5-25 \mu \mathrm{m}$ (characterization was carried out using wave numbers ranging 400-4000 $\left.\mathrm{cm}^{-1}\right)$. In practice IR spectra can be recorded using two different IR spectrometers: classic instruments double beam optical null electromagnetic radiation, and modern appliances irradiation pulses and Fourier transform (FTIR). Both IR spectrometers are based on same operating principle: electromagnetic radiation in IR emitted from a light source is passed through sample and then analyzed emerging radiation whose intensity appears altered by interaction with molecules of organic compound. FTIR analysis showed specific absorption bands acids and esters $(\mathrm{C}=\mathrm{O}) 1745 \mathrm{~cm}^{-1}$ at $1800-1500 \mathrm{~cm}^{-1}$ and $1500-1200 \mathrm{~cm}^{-1}$ specific carotenoids and $1726 \mathrm{~cm}^{-1}, 1072 \mathrm{~cm}^{-1}(\mathrm{C}-$ $\mathrm{OH}$ groups of oligosaccharide or flavonoid compounds residues) and $1638 \mathrm{~cm}^{-1}$ (protein) in case of phenolic compounds $[10,11]$.

Using chemometric analysis (PCA) of data from main FTIR bands were identified in extracts of carotenoids and phenolic compounds that contribute to classification of samples. Non-target analysis based on infrared spectroscopy (FTIR) provided much better data that led to significant discrimination of samples of seeds of $G$. aparine versus chromatographic analysis.

In conclusion, to discriminate differences between varieties of seeds of G. aparine, based on their metabolic fingerprints of two techniques used (FTIR and HPLC) had precision, accuracy and speed to achieve proper identification and authentication of biological varieties $G$. aparine. The methodology (called generic plant metabolomics) falls into category of biotechnology "omics" that are currently applied applicative purposes, to assess quality, authenticity, traceability and food safety issues that have particular relevance for chemotaxonomy, food science and industry food, pharmacy and medicine.

\section{References}

1. Veda S, Platel K, Srinivasan K (2008) Influence of food acidulants and antioxidant spices on the bioaccessibility of beta-carotene from selected vegetables. J Agric Food Chem 56: 8714-8719.

2. Bauer G, Klein MC, Gorb SN, Speck T, Voigt D, et al. (2011) Always on the bright side: the climbing mechanism of Galium aparine. Proc Biol Sci 278: 2233-2239.

3. Leonelli S, Smirnoff N, Moore J, Cook C, Bastow R (2013) Making open data work for plant scientists. J Exp Bot 64: 4109-4117.

4. Wang X, Zhang A, Yan G, Sun W, Han Y, et al. (2013) Metabolomics and proteomics annotate therapeutic properties of geniposide: targeting and regulating multiple perturbed pathways. PloS one Public Library of Science 8: e71403.

5. Kraft M, Kuglitsch R, Kwiatkowski J, Frank M, Grossmann K (2007) Indole-3acetic acid and auxin herbicides up-regulate 9-cis-epoxycarotenoid dioxygenase gene expression and abscisic acid accumulation in cleavers (Galium aparine): interaction with ethylene. J Exp Bot 58: 1497-1503.

6. Barbat C, Rodino S, Petrache S, Butu M, Butnariu M (2013) Microencapsulation of the allelochemical compounds and study of their release from different products. Digest Journal of Nanomaterials and Biostructures 8: 945-953. 
7. Tangkhavanich $B$, Kobayashi $T$, Adachi S (2012) Properties of rice straw extract after subcritical water treatment. Biosci Biotechnol Biochem 76: 1146-1149.

8. Lu X, Rasco BA, Kang DH, Jabal JM, Aston DE, et al. (2011) Infrared and Raman spectroscopic studies of the antimicrobial effects of garlic concentrates and diallyl constituents on foodborne pathogens. Anal Chem 83: 4137-4146.

9. Wilson MB, Spivak M, Hegeman AD, Rendahl A, Cohen JD (2013) Metabolomics reveals the origins of antimicrobial plant resins collected by honey bees. PLoS One 8: e77512.

10. Mari A, Lyon D, Fragner L, Montoro P, Piacente S, et al. (2013) Phytochemical composition of Potentilla anserina L. analyzed by an integrative GC-MS and LC-MS metabolomics platform. Metabolomics 9: 599-607.

11. Samfira I, Butnariu M, Rodino S, Butu M (2013) Structural investigation of Mistletoe plants from various Hosts exhibiting diverse lignin phenotypes. Digest Journal of Nanomaterials and Biostructures 8: 1679-1686. 